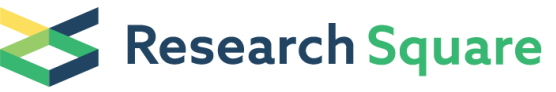 \\ Preprints are preliminary reports that have not undergone peer review. \\ They should not be considered conclusive, used to inform clinical practice, or referenced by the media as validated information.
}

\section{Telemedical care improves quality of life in patients with schizophrenia and bipolar disorder. Results of a randomized controlled trial}

Ulrike Stentzel ( $\nabla$ ulrike.stentzel@uni-greifswald.de)

Institute for Community Medicine, University Medicine Greifswald https://orcid.org/0000-0002-97238677

\section{Neeltje van den Berg}

Universitatsmedizin Greifswald, Institut für Community Medicine

\section{Lara N. Strobel}

Universitatsklinikum Greifswald Klinik und Poliklinik fur Psychiatrie und Psychotherapie Bereich

Greifswald

\section{Josephine Schulte}

Universitatsmedizin Greifswald

Jens M. Langosch

Krankenhaus Bethanien gGmbH

\section{Wolfgang Hoffmann}

Universitätsmedizin Greifswald, Institut für Community Medicine

Hans J. Grabe

Universitatsmedizin Greifswald, Klinik und Poliklinik für Psychiatrie und Psychotherapie

\section{Research article}

Keywords: Telemedical care, telemedicine, Schizophrenia, Bipolar disorder, Quality of life, WHOQOL-BREF, Randomized controlled trial

Posted Date: May 6th, 2020

DOI: https://doi.org/10.21203/rs.2.24510/v2

License: (c) (1) This work is licensed under a Creative Commons Attribution 4.0 International License. Read Full License

Version of Record: A version of this preprint was published at BMC Psychiatry on June 29th, 2021. See the published version at https://doi.org/10.1186/s12888-021-03318-8. 


\section{Abstract}

Background: Schizophrenia and bipolar disorder are serious psychiatric disorders with a high disease burden, many years lived with disability and a high level of risk of relapses and re-hospitalisations. Besides, both diseases are often accompanied with a reduced quality of life. A low quality of life is one predictor for relapses. This study examines whether a telemedical care programme can improve quality of life.

Methods: "Post stationary telemedical care of patients with severe psychiatric disorders" (Tecla) is a prospective controlled randomised intervention trial to implement and evaluate a telemedical care concept for patients with schizophrenia and bipolar disorder. Participants were randomised in an intervention or a control group. The intervention group received telemedical care including regular, individualised telephone calls and SMS messages. The quality of life was measured with the German version of WHOQOL-BREF. Effects of telemedicine on quality of life after 6 months were analysed using ttests to compare the intervention with the control group. Participants also evaluated the telemedical care program based on a short standardised interview.

Results: 118 participants were recruited, thereof $57.6 \%$ men $(n=68)$. Participants were 43 years old on average (SD) 13)). The IG showed higher QoL scores than the control group (CG) 6 months after the baseline for the WHOQOL total sum score (t-test $(\mathrm{Cl}) 93.1$ (92.4-93.8) vs $89.7(88.8-90.6), p<0.0001)$ and for 4 of 5 domains: global 62.0 (60.9-63.0) vs. 56.8 (55.6-58.1), $p<0.0001$; physical health 63.8 (63.0$64.7)$ vs. 59.6 (58.5-60.6), $p<0.0001$; psychological 60.9 (60.0-61.9) vs. 56.4 (55.1-57.6), $p<0.0001$; environment 70.8 (70.1-71.6) vs. $67.5(66.7-68.3), p<0.0001)$.

Conclusion: The Tecla telemedical care concept has led to improvements in quality of life for patients with severe psychiatric disorders. It provides a low-threshold and suitable component of psychiatric treatment.

Trial registration: German Clinical Trials Register, DRKS00008548, registered 21 May 2015 retrospectively registered, https://www.drks.de/drks_web/setLocale_EN.do

\section{Introduction}

Mental illnesses have a high disease burden and the number of days with limitations is 3 times higher in patients with mental illnesses than healthy people [1]. The course of mental diseases is often chronic [2]. Schizophrenia and bipolar disorder are among the most serious psychiatric disorders. Schizophrenia is one of the ten diseases with the highest number of years lived with disability (YLD) [3]. Relapses and rehospitalisation are frequent in patients with schizophrenia and bipolar disorder $[4,5]$. Both diseases are often accompanied with a distinct impairment of social and professional life management and thus result in a lasting reduced quality of life [3, 6-8]. The World Health Organization Quality of Life (WHOQOL) Group defined quality of life as "individuals' perceptions of their position in life in the context of the culture and value systems in which they live and in relation to their goals, expectations, standards and 
concerns." [9]. All aspects of life, which means physical, social, environmental and psychological aspects, affect wellbeing and satisfaction [6].

Schizophrenia and bipolar disorder patients show similar quality of life levels [6]. A low level of quality of life is a predictor for relapses [10]. Akvardar et al. showed that the improvement of quality of life is one important part of treating psychiatric disorders [7]. Hence, quality of life is an important factor and must be a target for gaining a good or at least stable state of mental health $[7,11]$.

Telemedicine has the potential to improve healthcare for patients within the mental health spectrum. Positive effects were shown on patients with anxiety and depression [12] and on medication adherence in patients with schizophrenia and bipolar disorder [13].

This paper reports results regarding quality of life from a prospective controlled randomised intervention trial called "Post stationary telemedical care of patients with severe psychiatric disorders" (Tecla). Tecla's objective was the implementation and evaluation of a telemedical care concept for patients with schizophrenia, schizoaffective disorder and bipolar disorder. It addressed different problems in treatment and everyday life management [14]. The primary outcome was medication adherence, which was positively influenced by the telemedical care concept [13]. This article aims to investigate the effects of the telemedical care concept on quality of life in patients with schizophrenia, schizoaffective disorder and bipolar disorder. The hypothesis is that the participants in the intervention group, which received additional telemedical care, had a better quality of life than participants in a control group, which received its usual care for six months after the baseline.

\section{Methods}

\section{Patient sample and data}

Data was retrieved from the prospective controlled randomised intervention trial Tecla. The inclusion criteria of Tecla were a medical diagnosis of any form of schizophrenia (ICD-10 F20), schizoaffective disorders (ICD-10 F25) or bipolar disorders (ICD-10 F31) and an age $\geq 18$ years. The diagnoses were extracted from the patient files. The exclusion criteria were prior scheduled inpatient treatment within the next six months and lack of ability to be contacted by mobile phone. The participants were recruited shortly before their discharge from day-care hospitals or open or locked inpatient wards from three psychiatric departments in Western-Pomerania, a federal state in the North East part of Germany.

Tecla has been approved by the Ethics Committee of the University Medicine Greifswald (BB 122/14) and was registered on the German Clinical Trials Register (date 2015 05\21, DRKS00008548). A comprehensive description of the study protocol for the Tecla study was published by Stentzel et al. [14].

\section{Randomisation}

The participants were randomized in the intervention or control group after the baseline assessment. A scientist who was neither involved in the recruitment nor in the baseline assessment performed the 
allocation to the groups using a random allocation (block randomisation) on a blind basis. The listing of the two groups was irregular. The participants with chronic conditions were assigned to the next entry in the randomisation list.

\section{Telemedical intervention}

Participants were individually randomised in the intervention group and control group. Both groups received care as usual in the outpatient facilities (outpatient psychiatric / psychotherapeutic practices or psychiatric institutional outpatient departments). The intervention group received regular telephone calls every two weeks and standardised and individualised text messages every week. An example of an individualised text message is provided in Figure 1. Qualified nurses who specialise in telemedical care conducted the regular telephone calls. The nurses are part of regular meetings at one of the psychiatric institutions' outpatient departments and day-care hospital. They received training in how to use the documentation system and have completed appropriate psychiatric/psychotherapeutic education programmes. The telemedical conversation was conducted on the basis of eCRFs in a computer-aided documentation system in accordance with the current standards for data security and data privacy [15, 16]. The standardised conversation contained a structured standardised part and an individualised part. The structured standardised part of the telephone calls included suicidal tendencies, changes in the medication regime, medication adherence and medication side effects (study protocol published elsewhere [13]). The individualised part addressed selected topics of everyday life that the respective participant evaluated as important for themselves and their condition. The weekly text messages refer to actual and relevant events and themes in the daily life of the participants.

\section{Measures}

\section{WHOQOL-BREF}

Quality of life was measured with WHOQOL-BREF, the short version of the World Health Organization Quality of Life subjective instrument, which is designed for generic use [9, 17]. It assesses quality of life from a subjective perspective [7]. The short version, WHOQOL-BREF, has 26 items. Answers are given on 1-to-5-point Likert scales. The higher the total score, the better the patient's quality of life [17]. WHOQOL assesses different aspects of life that are relevant for quality of life [9]. The WHOQOL-BREF is based on four domains $[9,17]$ and one global value for general quality of life:

- Physical domain: pain, energy, sleep, mobility, activities, medication, work.

- Psychological domain: positive feelings, cognitions, self-esteem, body image, negative feelings, spirituality.

- Social relationships: personal relationships, social support, sex.

- Environment: safety and security, home environment, finance, health/social care, information, leisure, physical environment, transport.

- Global: overall quality of life, general health. 
The German version was used, which shows good internal consistence (Cronbach's $a>0.7$ for all domains) for the overall population as well as for patients with mental illnesses [18].

\section{Participants' evaluation of the telemedical care programme}

Participants in the intervention group were asked to evaluate the telemedical care at the end of their time participating in the study by answering the questions shown in Table 1.

Table 1: Interview questions and answers to assess the participants' acceptance and satisfaction

\begin{tabular}{|l|l|}
\hline Question: & How would you describe the telephone and text message contact during the last 6 months? \\
\hline Answer: & Very helpful - moderately helpful - not helpful - other (free text) - don't know - no answer \\
\hline Question: & Would you like to continue the telephone support in this form? \\
\hline Answer: & Yes - no - don't know - no answer \\
\hline Question: & $\begin{array}{l}\text { In the future, could this telephone support make contacts to doctors or psychologists less necessary or } \\
\text { perhaps partly replace them? }\end{array}$ \\
\hline Answer: & Yes - no - don't know - no answer \\
\hline Question: & Is there anything you would change or improve? \\
\hline Answer: & Yes - no - don't know - no answer and additional free text \\
\hline
\end{tabular}

\section{Statistical analysis}

The baseline characteristics were compared by group affiliation to identify any group differences at the baseline. We used t-tests for continuous variables and Chi-square tests for categorical variables. To analyse effects of the telemedical intervention on quality of life, the WHOQOL total score and each of the WHOQOL domains of the intervention group were compared with the scores of the control group after 6 months using t-tests.

The analyses were conducted with the intention-to-treat approach. For randomised clinical trials with missing data, the multiple imputation procedure is a valid method for handling missing data [19] and minimising possible biases [20]. However, a required condition for multiple imputation is that missing data is distributed completely at random (MCAR) or at random (MAR), whereas the method is less appropriate for data missing not at random (MNAR) [21]. After thorough inspection, we appraised the missing data as MAR. The proportion of missing values ranged from $11-17 \%$ (WHOQOL variables 12\%). 
Therefore, multiple imputation was used. To be able to reproduce the results, the random seed value was specified each time the analysis was performed [19]. Eighteen variables were included in the imputation model. Minimum and maximum values for score values were defined. Further details are documented in the supplementary information file. All statistical procedures were performed in SAS 9.4 (@) 2002-2012 by SAS Institute Inc., Cary, North Carolina, USA.).

\section{Results}

118 participants were recruited (see CONSORT flow diagram in Figure 2), thereof $57.6 \%$ men $(n=68)$. Participants were 43 years old on average (standard deviation (SD) 13). Baseline characteristics are shown in Table 2. Except for education, there was no significant difference between the intervention and control group at baseline. Participants in the intervention group had a higher level of education than participants in the control group. 104 diagnoses of schizophrenia and schizoaffective disorder (ICD-10 F2x.) and 48 bipolar disorder diagnoses (ICD-10 F3x.) were found. 21 patients had two to three diagnoses. Further details are documented in Table 1 in the supplement supplementary information file. 90 participants remained in the study until the six-month follow-up assessment. Of these, 79 participants completed the WHOQOL-BREF.

Table 2: Characteristics of the participants at baseline. The differences between the intervention and control group were analysed for categorical variables with $\mathrm{Chi}^{2}$ and for continuous variables with a $t$-test. 


\begin{tabular}{|c|c|c|c|c|}
\hline $\mathrm{Chi}^{2}$ & $\begin{array}{l}\text { Total } \\
\text { n (\%) }\end{array}$ & $\begin{array}{c}\text { Intervention } \\
\text { group } \\
\mathrm{n}(\%)\end{array}$ & $\begin{array}{c}\text { Control group } \\
\text { n (\%) }\end{array}$ & $\begin{array}{c}\mathrm{p}- \\
\text { value }\end{array}$ \\
\hline Participants & $\begin{array}{c}118 \\
(100)\end{array}$ & $58(49.2)$ & $60(50.8)$ & \\
\hline Female & $50(42.4)$ & $27(22.9)$ & $23(19.5)$ & 0.3664 \\
\hline Psychiatric disease* & & & & 0.4734 \\
\hline $\begin{array}{r}\text { Schizophrenia / Schizoaffective disorder (ICD- } \\
10 \text { F2x.) }\end{array}$ & $\begin{array}{c}104 \\
(68.4)\end{array}$ & $52(34.2)$ & $52(34.2)$ & \\
\hline Bipolar disorder (ICD-10 F3x.) & $48(31.6)$ & $21(13.8)$ & $27(17.8)$ & \\
\hline Education: & & & & 0.0002 \\
\hline$<10$ years & $32(32.3)$ & $6(6.1)$ & $26(26.3)$ & \\
\hline 10 years & $42(42.4)$ & $25(25.3)$ & $17(17.2)$ & \\
\hline$>10$ years & $25(25.3)$ & $17(17.2)$ & $8(8.1)$ & \\
\hline
\end{tabular}

Employment:

0.3483

$\begin{array}{rccc}\text { Not employed } & 98(85.2) & 45(39.1) & 53(46.1) \\ \text { Marginally employed } & 5(4.4) & 3(2.6) & 2(1.7) \\ \text { Employed } & 12(10.4) & 8(7.0) & 4(3.5)\end{array}$

Social living situation:

0.9299

$$
\text { Living alone } 56 \text { (51.4) } 27 \text { (24.8) } 29 \text { (26.6) }
$$

Living with spouse, partner or assisted living $53(48.6) \quad 26(23.9) \quad 27(24.8)$

\begin{tabular}{|c|c|c|c|c|}
\hline t-test & \multirow{2}{*}{$\begin{array}{c}\text { Total } \\
\text { mean } \\
(\mathrm{SD}) \\
42.9 \\
(13.0)\end{array}$} & \multirow{2}{*}{$\begin{array}{c}\begin{array}{c}\text { Intervention } \\
\text { group }\end{array} \\
\text { mean (95\% CI) } \\
43.9(40.5-47.4)\end{array}$} & \multirow{2}{*}{$\begin{array}{c}\text { Control group } \\
\text { mean (95\% } \\
\text { CI) } \\
42.0(38.6- \\
45.2)\end{array}$} & \multirow{2}{*}{$\begin{array}{c}\begin{array}{c}\text { p- } \\
\text { value }\end{array} \\
0.4099\end{array}$} \\
\hline age & & & & \\
\hline WHOQOL total score & $\begin{array}{c}87.2 \\
(14.0)\end{array}$ & $86.8(83.0-90.8)$ & $\begin{array}{c}87.6(83.6- \\
91.2)\end{array}$ & 0.7927 \\
\hline \multicolumn{5}{|l|}{ WHOQOL domains: } \\
\hline Global & $\begin{array}{c}49.3 \\
(21.0)\end{array}$ & $46.0(39.9-52.2)$ & $\begin{array}{c}52.4(46.8- \\
58.0)\end{array}$ & 0.1246 \\
\hline Physical health & $\begin{array}{c}56.3 \\
(16.7)\end{array}$ & $56.8(51.9-61.5)$ & $\begin{array}{c}55.8(51.2- \\
60.5)\end{array}$ & 0.7931 \\
\hline Psychological & $\begin{array}{c}56.3 \\
(17.2)\end{array}$ & $56.8(51.9-61.7)$ & $\begin{array}{c}55.8(51.1- \\
60.6)\end{array}$ & 0.7740 \\
\hline Social relationships & $\begin{array}{c}57.3 \\
(21.3)\end{array}$ & $53.7(48.0-59.3)$ & $\begin{array}{c}60.7(54.5- \\
66.9)\end{array}$ & 0.0938 \\
\hline Environment & $\begin{array}{c}66.1 \\
(15.2)\end{array}$ & $66.3(62.2-70.5)$ & $\begin{array}{c}65.9(61.5- \\
70.3)\end{array}$ & 0.8826 \\
\hline
\end{tabular}

\footnotetext{
*Higher overall numbers because some patients had both diagnoses, CI = confidence interval
} 
The results of the analysis of quality of life after six months are shown in Table 3. Both the total score and the separate domains show significant differences between the intervention group and control group. The intervention group showed higher quality of life scores between $3.3-5.2$ points for the global, physical health, psychological and environmental domain. On the other hand, the control group showed a significantly better score within social relationships.

Table 3: Analysis of differences in quality of life between the intervention and control group at the six-month-follow-up assessment using a t-test

\begin{tabular}{rccr}
\hline & $\begin{array}{c}\text { Intervention group } \\
\text { mean (95\% CI) }\end{array}$ & $\begin{array}{c}\text { Control group } \\
\text { mean (95\% CI) }\end{array}$ & p-value \\
\hline WHOQOL total score & $93.1(92.4-93.8)$ & $89.7(88.8-90.6)$ & $<0.0001$ \\
\hline WHOQOL domains: & & & \\
\hline Global & $62.0(60.9-63.0)$ & $56.8(55.6-58.1)$ & $<0.0001$ \\
\hline Physical health & $63.8(63.0-64.7)$ & $59.6(58.5-60.6)$ & $<0.0001$ \\
\hline Psychological & $60.9(60.0-61.9)$ & $56.4(55.1-57.6)$ & $<0.0001$ \\
\hline Social relationships & $58.2(57.3-59.1)$ & $61.1(59.9-62.3)$ & 0.0002 \\
\hline Environment & $70.8(70.1-71.6)$ & $67.5(66.7-68.3)$ & $<0.0001$ \\
\hline CI = confidence interval & & &
\end{tabular}

Results of sensitivity analyses are shown in the supplementary information file. Differences in the intervention group means between the baseline and six-month follow-up assessment without multiple imputation (MI) are shown in supplement Table 2 and with multiple imputation (MI) in the annex in Table 3. Differences in means between the intervention and control group at the six-month follow-up assessment without and with multiple imputation (MI) are shown in thesupplement t supplementary information file in Table 4.

The results of the evaluation of the telemedical programme by participants of the intervention group are shown in Figure 3. Participants perceived the telemedical care mostly as moderately helpful to very helpful (97.5\%, Figure 3A). The majority would like to continue the telemedical care (73.2\%, Figure 3B). A minority can even imagine that the tele medical care could make contact with doctors or psychologists less necessary or perhaps partly replace this contact (34.2\%, Figure 3C).

\section{Discussion}

Quality of life is a major treatment goal for patients with psychiatric disorders [7, 11, 23]. A low-threshold telemedical care programme consisting of regular telephone calls and SMS messages was able to improve quality of life compared to a control group in almost all aspects. These findings are in line with another study that also investigated a mobile health (mHealth) approach. Ben-Zeev (et. al) compared the 
mHealth intervention FOCUS with a widely used group self-management intervention called WRAP [24]. Quality of life was investigated as one of the secondary outcomes. The FOCUS participants showed significant improvements between the baseline and the six-month follow-up assessment. Even though the FOCUS intervention substantially differs from Tecla, the mode of administration via information and communication technologies is similar. The general feasibility, acceptance and efficiency of electronic Health (eHealth) and mHealth interventions for people with serious mental illnesses is proven by several other studies [25-27].

However, the WHOQOL was proven to be an adequate tool for assessing quality of life in different cultures and population groups $[28,29]$. Therefore, in this study, we adopted a generic tool [6], that can be broadly applied for assessing quality of life in different cultures and population groups [30,31]. The WHOQOL-BREF is less affected by disease-related factors [17] and has been applied in patients with schizophrenia with good reliability and validity [31, 32], even in psychotic stages, in patients on medication and in patients with relatively low education levels [7]. Kim et al. compared patients' assessments of their own quality of life using WHOQOL-BREF with assessments of proxies (such as family members, caregivers) and found a moderate to good correlation between both assessments of the patients' quality of life [8].

Even though schizophrenia and bipolar disorder are different diseases, there are similarities between them, such as quality of life. Both diseases showed similar scores for the WHOQOL-BREF domains in previous studies [11,33]. In this study, the baseline characteristics showed no differences between the diagnostic groups (see Table 2). Hence, both diseases were analysed together.

A strength of this study is the use of the usual care setting with few inclusion and exclusion criteria. Consequently, the results are likely to be transferable to a large part of the patient group and daily regular medical care. In this regular care setting, the study was conducted with an RCT design. Multiple imputation was performed to strengthen the validity.

The baseline assessment showed a significant difference between the two groups with respect to the level of education. Participants in the intervention group had a higher level of education compared to participants in the control group. A scientist performed the allocation to the groups using random allocation (block randomisation) after the baseline assessment on a blind basis. However, the baseline characteristics showed similar values for all WHOQOL domains for both groups (see Table 2). In fact, the intervention group even had a slightly lower WHOQOL total score values. The intervention was largely standardised. Furthermore, the loss to follow-up was identical in both groups (see Figure 2). Therefore, a systematic bias seems unlikely. The proportion of loss to follow-up at the six-month follow-up assessment was $2 \%$ in the invention group and $23 \%$ in the control group. Due to the size of the dropout rates, there may be an attrition bias [34, 35], but threshold levels for acceptable dropout levels are not determined in guidelines yet [35]. Furthermore, distinct patient groups might require different levels. Due to the almost identical rates and the difficult patient groups, we deem that the potential bias may be low. In addition, the loss to follow-up is similar to other reported dropout rates in the patient groups being studied [31]. 
Diagnoses were extracted from the patients' files from the three recruiting psychiatric departments. In several cases, a clear diagnosis had not yet been finalised by the physicians treating the patient. Therefore, several diagnoses were sometimes applicable here.

Medication and its side effects could possibly affect patients' quality of life [11] and knowledge of this would have been useful, but these aspects were not included here. However, it is a relevant issue. Therefore, the influence of medication on various data collected within the Tecla study, including the quality of life aspect, is currently being evaluated.

\section{Conclusion}

Every aspect that may help to stabilise the patient and avoid hospitalisation should be considered during treatment. The telemedical intervention shown here is a low-threshold care concept that has the potential to improve the care situation of patients with severe psychiatric illnesses. Schulze et al. previously demonstrated that Tecla improved medication adherence [13]. In this study, we looked at Tecla's impact on the overall quality of life of the participants. Quality of life relates to the personal, subjective perspective on life and has a high degree of relevance for the patients. The Tecla telemedical care intervention addressed both general and individual issues of daily life for the participants. The intervention was successfully integrated in the usual care given to the patients.

The Tecla intervention has considerable potential as a complementary element in addition to the patient's usual care and may help to avoid treatment gaps or re-hospitalisation. Therefore, it should be considered a suitable and appropriate treatment component for patients with severe psychiatric illnesses.

\section{List Of Abbreviations}




\begin{tabular}{ll} 
Cl & Confidence interval \\
\hline DF & Degree of freedom \\
\hline eCRF & Electronic Care Report Forms \\
\hline eHealth & Electronic health \\
\hline MAR & Missing at random \\
\hline MCAR & Missing completely at random \\
\hline MNAR & Missing not at random \\
\hline mHealth & Mobile health \\
\hline MI & Multiple imputation \\
\hline OR & Odds ratio \\
\hline SD & Standard deviation \\
\hline Tecla & $\begin{array}{l}\text { Study “Post stationary telemedical care of patients with severe psychiatric } \\
\text { disorders” }\end{array}$ \\
\hline WHOQOL & World Health Organization Quality of Life \\
\hline WHOQOL- & World Health Organization Quality of Life, short form with 26 items \\
BREF & Years of life lived with disability \\
\hline YLD &
\end{tabular}

\section{Declarations}

\section{Ethics approval and consent to participate}

Tecla is approved by the Ethics Committee of University Medicine Greifswald (BB 122/14). The committee stated that the majority of the members of the committee concluded that there are no ethical and legal concerns regarding the implementation of the study and the committee therefore approves the proposal. Tecla is retrospectively registered under 2015\05\21 on the German Clinical Trials Register (DRKS00008548). All patients had to sign an informed consent form to participate. If appropriate, legal guardians or representatives were informed about the participation. All guardians or representatives indicated that the patients were capable of providing ethical consent to participate.

\section{Consent for publication}

Not applicable.

\section{Availability of data and material}


The datasets used and/or analysed during the current study are available from the corresponding author on reasonable request.

\section{Competing interests}

The authors declare that they have no competing interests.

\section{Funding}

This study is funded by the Damp Foundation (Damp Stiftung c/o NGEG $\mathrm{mbH}$ ) after a peer review process. The Damp Foundation supports medical research and teaching, social projects and the education of young professionals in the medical profession in the German federal states of SchleswigHolstein, Hamburg and Western-Pomerania. The Damp Stiftung only financed the study. The study design, data collection, analysis, data interpretation and writeup of the manuscript were carried out independently from the funding body.

\section{Authors' contribution}

NvdB, HJG and WH designed the study. LNS, HJG, JS, JML and NvdB participated in the coordination of the patient recruitment. US and LNS coordinated the study. US conducted the statistical calculation with support from JS. US drafted the manuscript. WH provided comprehensive feedback on an early draft. All authors read and approved the final manuscript.

\section{Acknowledgements}

We acknowledge support for the Article Processing Charge from the DFG (German Research Foundation, 393148499) and the Open Access Publication Fund of the University of Greifswald.

\section{References}

1. Jacobi F, Höfler M, Strehle J, Mack S, Gerschler A, Scholl L, Busch MA, Maske U, Hapke U, Gaebel W et al: Mental disorders in the general population. Study on the health of adults in Germany and the additional module mental health (DEGS1-MH). In: Nervenarzt. 2014: 77-87.

2. Vibha P, Saddichha S, Khan N, Akhtar S: Quality of life and marital adjustment in remitted psychiatric illness: an exploratory study in a rural setting. The Journal of nervous and mental disease 2013, 201(4):334-338.

3. Gaebel W, Wölwer W: Schizophrenie. In. Berlin: Robert Koch-Institut; 2010.

4. Emsley R, Chiliza B, Asmal L, Harvey BH: The nature of relapse in schizophrenia. BMC Psychiatry 2013, 13:50.

5. Radua J, Grunze H, Amann BL: Meta-Analysis of the Risk of Subsequent Mood Episodes in Bipolar Disorder. Psychotherapy and psychosomatics 2017, 86(2):90-98. 
6. Amini H, Sharifi V: Quality of life in bipolar type I disorder in a one-year followup. Depression research and treatment 2012, 2012:860745.

7. Akvardar Y, Akdede BB, Ozerdem A, Eser E, Topkaya S, Alptekin K: Assessment of quality of life with the WHOQOL-BREF in a group of Turkish psychiatric patients compared with diabetic and healthy subjects. Psychiatry and clinical neurosciences 2006, 60(6):693-699.

8. Kim EJ, Song DH, Kim SJ, Park JY, Lee E, Seok JH, Jon DI, Cho HS: Proxy and patients ratings on quality of life in patients with schizophrenia and bipolar disorder in Korea. Quality of life research : an international journal of quality of life aspects of treatment, care and rehabilitation 2010, 19(4):521-529.

9. WHO: Developement of the World Health Organization WHOQOL-BREF quality of life assessment. The WHOQOL Group. Psychol Med 1998, 28(3):551-558.

10. Boyer L, Millier A, Perthame $E$, Aballea $S$, Auquier $P$, Toumi M: Quality of life is predictive of relapse in schizophrenia. BMC Psychiatry 2013, 13:15.

11. Brissos S, Dias VV, Carita Al, Martinez-Aran A: Quality of life in bipolar type I disorder and schizophrenia in remission: clinical and neurocognitive correlates. Psychiatry research 2008, 160(1):55-62.

12. van den Berg N, Grabe HJ, Baumeister SE, Freyberger HJ, Hoffmann W: A Telephone- and Text Message-Based Telemedicine Concept for Patients with Mental Health Disorders: Results of a Randomized Controlled Trial. Psychotherapy and psychosomatics 2015, 84(2):82-89.

13. Schulze LN, Stentzel U, Leipert J, Schulte J, Langosch J, Freyberger HJ, Hoffmann W, Grabe HJ, van den Berg N: Improving Medication Adherence With Telemedicine for Adults With Severe Mental Illness. Psychiatric services (Washington, DC) 2019:appips201800286.

14. Stentzel U, Grabe HJ, Strobel L, Penndorf P, Langosch J, Freyberger HJ, Hoffmann W, van den Berg N: Tecla: a telephone and text-message based telemedical concept for patients with severe mental health disorders-study protocol for a controlled, randomized, study. BMC Psychiatry 2015, 15:273.

15. Meyer J, Fredrich D, Piegsa J, Habes M, van den Berg N, Hoffmann W: A mobile and asynchronous electronic data capture system for epidemiologic studies. Computer methods and programs in biomedicine 2013, 110(3):369-379.

16. Bialke M, Penndorf P, Wegner T, Bahls T, Havemann C, Piegsa J, Hoffmann W: A workflow-driven approach to integrate generic software modules in a Trusted Third Party. Journal of translational medicine 2015, 13:176.

17. Skevington SM, Lotfy M, O'Connell KA: The World Health Organization's WHOQOL-BREF quality of life assessment: psychometric properties and results of the international field trial. A report from the WHOQOL group. Quality of life research : an international journal of quality of life aspects of treatment, care and rehabilitation 2004, 13(2):299-310.

18. Angermeyer MC, Kilian R, Matschinger H: WHOQOL-100 und WHOQOL-BREF - Handbuch für die deutschsprachige Version der WHO Instrumente zur Erfassung von Lebensqualität: Hogrefe-Verlag; 2000. 
19. Jakobsen JC, Gluud C, Wetterslev J, Winkel P: When and how should multiple imputation be used for handling missing data in randomised clinical trials - a practical guide with flowcharts. BMC medical research methodology 2017, 17(1):162-162.

20. Sterne JA, White IR, Carlin JB, Spratt M, Royston P, Kenward MG, Wood AM, Carpenter JR: Multiple imputation for missing data in epidemiological and clinical research: potential and pitfalls. $B M J$ 2009, 338:b2393.

21. Harel O, Mitchell EM, Perkins NJ, Cole SR, Tchetgen Tchetgen EJ, Sun B, Schisterman EF: Multiple Imputation for Incomplete Data in Epidemiologic Studies. Am J Epidemio/ 2018, 187(3):576-584.

22. Moher D, Schulz KF, Altman D: The CONSORT statement: revised recommendations for improving the quality of reports of parallel-group randomized trials. Jama 2001, 285(15):1987-1991.

23. Dong M, Lu L, Zhang L, Zhang YS, Ng CH, Ungvari GS, Li G, Meng X, Wang G, Xiang YT: Quality of Life in Schizophrenia: A Meta-Analysis of Comparative Studies. The Psychiatric quarterly 2019, 90(3):519-532.

24. Ben-Zeev D, Brian RM, Jonathan G, Razzano L, Pashka N, Carpenter-Song E, Drake RE, Scherer EA: Mobile Health (mHealth) Versus Clinic-Based Group Intervention for People With Serious Mental Illness: A Randomized Controlled Trial. Psychiatric services (Washington, DC) 2018, 69(9):978-985.

25. Ben-Zeev D, Brenner CJ, Begale M, Duffecy J, Mohr DC, Mueser KT: Feasibility, acceptability, and preliminary efficacy of a smartphone intervention for schizophrenia. Schizophrenia bulletin 2014, 40(6):1244-1253.

26. Berrouiguet S, Baca-Garcia E, Brandt S, Walter M, Courtet P: Fundamentals for Future Mobile-Health (mHealth): A Systematic Review of Mobile Phone and Web-Based Text Messaging in Mental Health. Journal of medical Internet research 2016, 18(6):e135.

27. Naslund JA, Marsch LA, McHugo GJ, Bartels SJ: Emerging mHealth and eHealth interventions for serious mental illness: a review of the literature. Journal of mental health (Abingdon, England) 2015, 24(5):321-332.

28. Atkinson $\mathrm{M}$, Zibin $\mathrm{S}$, Chuang $\mathrm{H}$ : Characterizing quality of life among patients with chronic mental illness: a critical examination of the self-report methodology. The American journal of psychiatry 1997, 154(1):99-105.

29. Gazalle FK, Frey BN, Hallal PC, Andreazza AC, Cunha AB, Santin A, Kapczinski F: Mismatch between self-reported quality of life and functional assessment in acute mania: a matter of unawareness of illness? Journal of affective disorders 2007, 103(1-3):247-252.

30. Oliveira SE, Carvalho H, Esteves F: Toward an understanding of the quality of life construct: Validity and reliability of the WHOQOL-Bref in a psychiatric sample. Psychiatry research 2016, 244:37-44.

31. Olusina AK, Ohaeri JU: Subjective quality of life of recently discharged Nigerian psychiatric patients. Social psychiatry and psychiatric epidemiology 2003, 38(12):707-714.

32. Mas-Expósito L, Amador-Campos JA, Gómez-Benito J, Lalucat-Jo L: The World Health Organization Quality of Life Scale Brief Version: a validation study in patients with schizophrenia. Quality of Life Research 2011, 20(7):1079-1089. 
33. Yen $\mathrm{CF}$, Cheng $\mathrm{CP}$, Huang $\mathrm{CF}$, Yen JY, Ko CH, Chen CS: Quality of life and its association with insight, adverse effects of medication and use of atypical antipsychotics in patients with bipolar disorder and schizophrenia in remission. Bipolar disorders 2008, 10(5):617-624.

34. Brueton VC, Tierney J, Stenning S, Harding S, Meredith S, Nazareth I, Rait G: Strategies to improve retention in randomised trials. The Cochrane database of systematic reviews 2013(12):Mr000032.

35. Szymczyńska P: Retention of Patients with Schizophrenia in Complex Intervention Trials: Patterns, Issues, and Practices. Queen Mary University of London; 2018.

\section{Figures}

\section{Study nurse:}

Good morning Mrs. $X X$, this is Sister $Y Y$ from the telephone study from Greifswald. Were you still in the "Klex" yesterday for choir practice and how did you like it? could you get the medicine from the pharmacy yesterday? I would appreciate an answer from you. Sunny greetings sister $Y Y$

\section{Participant:}

Good morning, yes I was in the "Klex"

yesterday and I picked up the pills today and

took them. Right now I'm sitting at my GP's

getting an ECG. Love $X X$

Figure 1

Example for an individualized text message contact between study nurse and participant 


\section{Enrollment}

Patients recruited

$$
n=118
$$

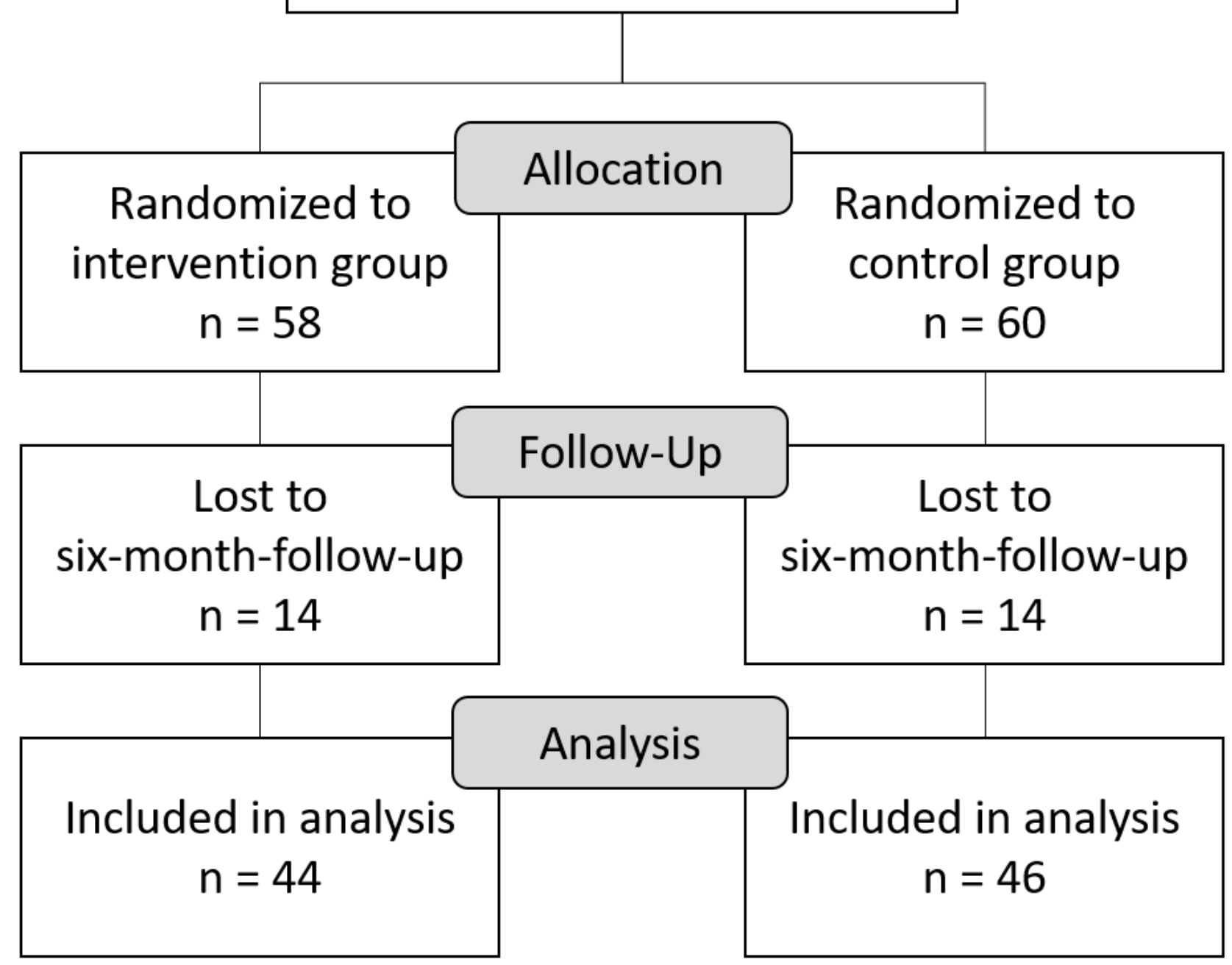

Figure 2

CONSORT flow diagram [22] 


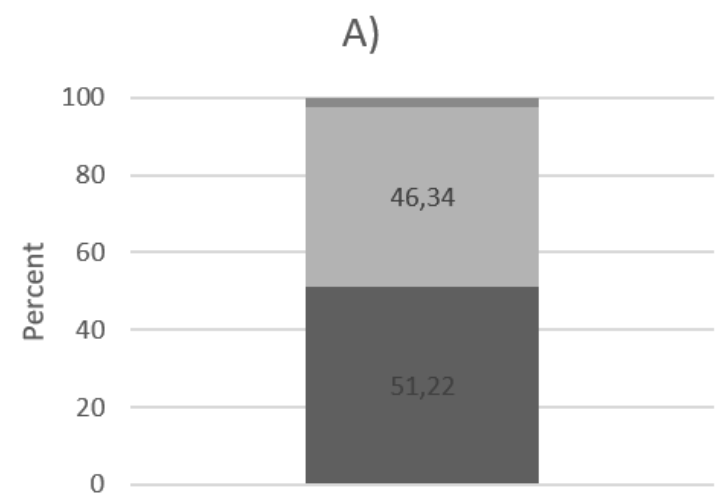

How would you describe the telephone support during the last six months?

- Veryhelpful Moderately helpful al don't know

B)

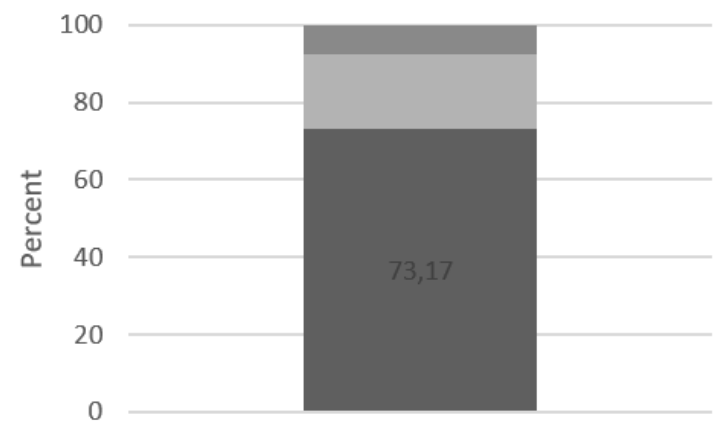

Would you like to continue the telephone support in this form?

- Yes No aldon't know

C)

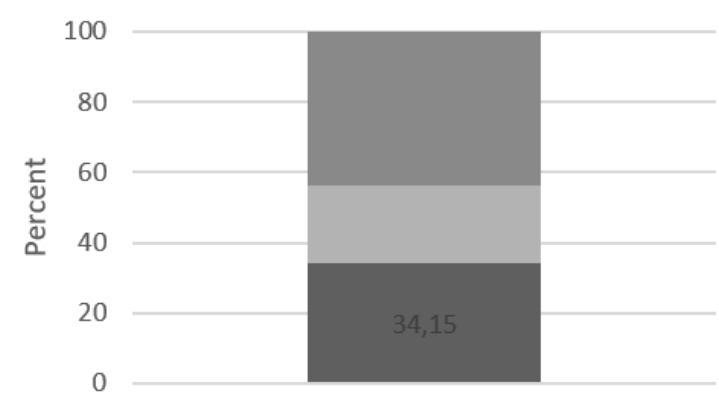

In the future, could this telephone support make contacts to doctors or psychologists less necessary or perhaps partly replace them?

-Yes No aldon't know

\section{Figure 3}

Subjective evaluation of received telemedicine care by the participants of the intervention group

\section{Supplementary Files}

This is a list of supplementary files associated with this preprint. Click to download. 
- CONSORT2010ChecklistTecla.doc

- SupplementMultiplelmputation.docx 